\title{
OFFLINE AND ONLINE MODELLING OF SWITCHED RELUCTANCE MOTOR BASED ON RBF NEURAL NETWORKS
}

\author{
Jun Cai — Zhiquan Deng *
}

\begin{abstract}
Due to the highly nonlinearity of the flux-linkage characteristics of Switched Reluctance Motor drives (SRM), accurately modelling is cumbersome. In this paper, the offline- trained and the online-trained Radial Basis function (RBF) neural network model are proposed for estimating the SRM flux-linkage under running conditions. To investigate the performance of the modelling schemes, the simulation and experiments have been implemented in a $12 / 8$ structure SRM prototype. The results show that the online-trained model exhibits much better estimation accuracy and robustness than the offline-trained model. Thus, the online-trained RBF model is more suitable for SRM performance prediction and analyzing.
\end{abstract}

K e y w or d s: offline modelling, online modelling, RBF neural network, flux-linkage, switched reluctance motor (SRM)

\section{INTRODUCTION}

Switched Reluctance Motor (SRM) drives are drawing great attention for variable speed motor drives due to its own advantages, such as simple structure, reliable power converter, good speed regulation performance and good fault-tolerant capacity. These drives are suitable for applications such as electric vehicles, aircraft starter/generators systems, mining drives, etc..

Accurately modelling of the flux linkage characteristics is crucial for optimal controls and performance predictions. However, due to its double salient structure, the magnetic characteristics are highly nonlinear. The fluxlinkage appears to be a complex nonlinear function of both the phase current and rotor position, which makes it cumbersome to model. In past literatures, numerous modelling approaches such as analytical models [1,2], look-up tables [3], fuzzy logics [4] and neural networks [5] based methods have been reported. As for the analytical based models, they can simplify the analysis of the machine. However, they always suffer from lack of accuracy and the complexity. The look-up tables based method is an intuitive approach. However, the three-dimensional lookup table requires huge memory space to store, and powerful computation capability of the controllers. Modelfree algorithms such as fuzzy logics and neural networks are inherently suitable for modelling systems with highly nonlinearity, which are drawing great attentions recently. However, the experimental results of these schemes are seldom discussed in past literatures. The fuzzy logic based and the ANN based offline-trained model are used for sensorless control of SRM in [4] and [5] respectively. The online modelling scheme for SRM is investigated firstly in [6], a 2-D BSNN is proposed for online modelling of SRM. However, the modelling performance between online and offline models are not compared in experiments.

The RBF network not only possesses good locally approximating capability, but also the optimal approximat- ing performance. The training process is simple, while the convergence rate is quite fast, it is no doubt that RBF is one of the best modelling algorithm for SRM modelling. In this paper, the RBF neural network based offline model and online model are proposed. The simulation and experimental results are presented and compared for validation.

\section{FUNDAMENTAL OF THE OFFLINE AND ONLINE MODELLING SCHEMES}

\subsection{Obtaining the flux linkage characteristics data}

Due to its double salient structure, the flux-linkage appears to be a complex nonlinear function of both the phase current and rotor position, which can be described by

$$
\Psi=\Psi(i, \theta)
$$

where $\Psi$ is flux linkage, $i$ is phase current, and $\theta$ is rotor position.

Accurately calculating the flux linkage characteristics is crucial for improving the accuracy and generalization ability of the RBF based model. In order to get better calculation results, the 3-D Finite Element Method (FEM) based on double scalar magnetic potential method (DSMP) is utilized to calculate the magnetic field of the SRM prototype. The boundary value problem can be given by

$$
\begin{aligned}
\nabla \cdot \mu \nabla \psi & =0, & \left.\frac{\partial \phi}{\partial n}\right|_{S_{2}} & =0, \\
\nabla \cdot \mu_{0} \nabla \phi & =0, & & -\mu \frac{\partial \psi}{\partial n}=\mu_{0} H_{s n}-\mu_{0} \frac{\partial \varphi}{\partial n}, \\
\left.\psi\right|_{S_{1}} & =0, & & \\
\left.\frac{\partial \psi}{\partial n}\right|_{S_{2}} & =0, & \varphi_{M}-\varphi_{N} & =\psi_{M}-\psi_{N}+\int_{N}^{M} H_{s t} \mathrm{~d} t
\end{aligned}
$$

\footnotetext{
* College of Automation Engineering, Nanjing University of Aeronautics and Astronautics, Nanjing, 210016, China, cainuaa3.com
} 


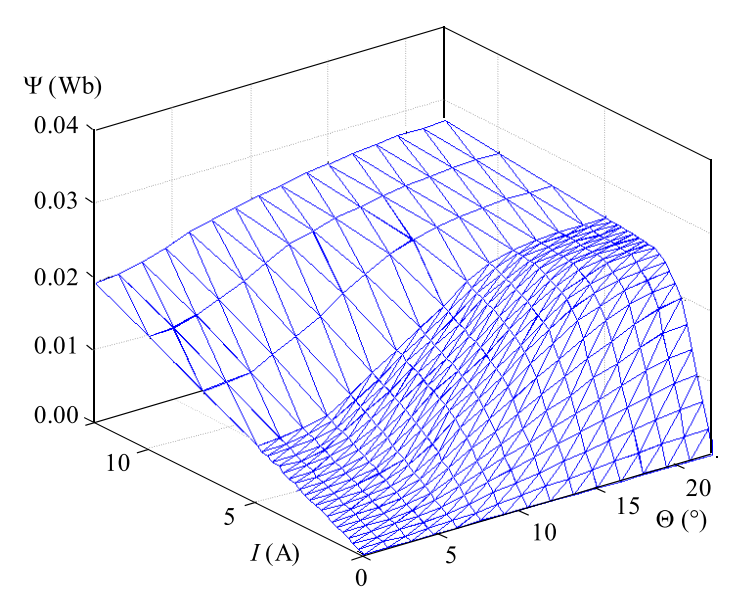

Fig. 1. The calculated flux linkage characteristics

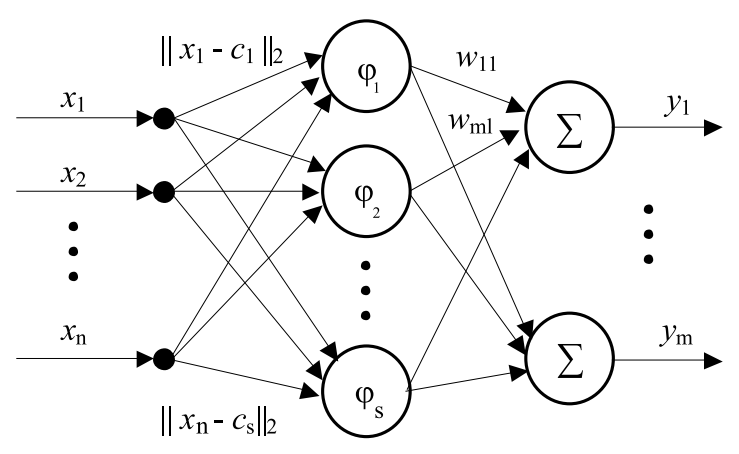

Fig. 2. The typical topology of an RBF neural network

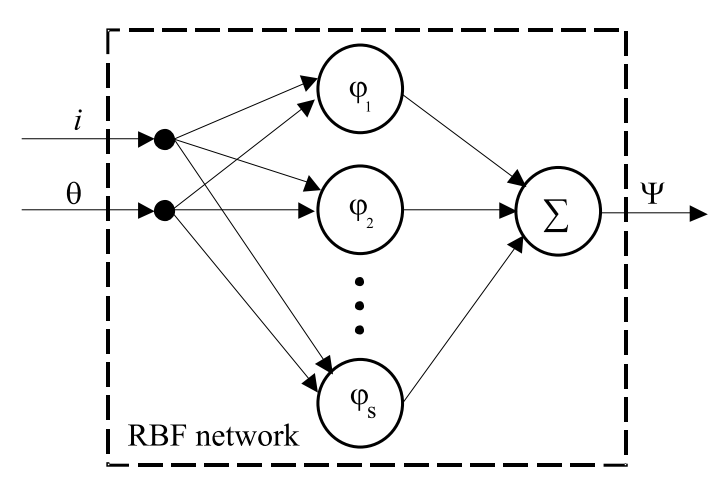

Fig. 3. The structure of offline RBF network based flux linkage model

where, $\psi$ is the full scalar magnetic potential; $\varphi$ is the partial scalar magnetic potential, $H_{s n}$ and $H_{s t}$ is the normal component and tangent component respectively, $S_{1}$ is the outer surface of stator core, and $S_{2}$ is the symmetrical face at the axial direction.

After acquiring of magnetic field distributions, the flux linkage characteristics can be calculated by enhanced in- cremental energy method (EIEM). The increment of magnetic co-energy $\Delta W_{C}$ can be expressed as

$$
\Delta W_{C}=\int B(\Delta H) \mathrm{d} V
$$

and the flux linkage is obtained by

$$
\Psi=\frac{\Delta W_{C}}{\Delta i}
$$

where $\Psi$ is the flux linkage, $B$ is the flux density, $\Delta H$ is magnetic field increment, $\mathrm{d} V$ is the volume differential, $\delta i$ is the current increment.

The 3-D computational result of flux linkage characteristic is shown in Fig. 1, where the rotor angular position $\theta$ is defined as $\theta=0^{\circ}$ at the unaligned position and $\theta=22.5^{\circ}$ at the aligned position.

\subsection{Modelling the flux linkage characteristics based on RBF neural networks}

\subsubsection{The RBF neural network}

As shown in Fig. 2, the RBF neural network is comprised of three layers: input layer, hidden layer and output layer. The input and output layers are presented with training pairs, each consisting of a vector from an input space and a desired network response. Through a defined learning algorithm, the error between the actual and desired response is minimized relative to some optimization criterion. As depicted in Fig. 2, the ith output note of the $\mathrm{RBF}$ network can be expressed as

$$
\begin{gathered}
y_{j}=\sum_{k=1}^{S} \varphi\left(\left\|x-c_{k}\right\|\right) w_{i k}, i=1,2, \ldots, m, \\
\phi_{k}\left(x_{j}\right)=\exp \left(-\frac{\left\|x_{j}-c_{j}\right\|^{2}}{\sigma_{k}^{2}}\right)
\end{gathered}
$$

where $x=\left[x_{1}, x_{2}, \ldots, x_{n}\right]^{\top}$ is an input vector; $n$ is the number of input node; $c_{k}$ is the RBF center, $k=$ $1,2, \ldots, S$, in which $S$ is the number of hidden nodes; $\left\|x-c_{k}\right\|$ denotes Euclidean distance between $c_{k}$ and $x$ vector; $\varphi_{k}($ ) is a Gaussian function of the $k$ th center, in which $\sigma_{k}$ is the Gussian variance; $w_{i k}$ is the weighting value between the $k$ th center and the $i$ th output node; and $m$ is the number of output nodes.

\subsubsection{Training algorithm}

In this paper, the centers and width of the RBF nodes are determined by the standard k-means clustering algorithm, whereas the output weights are normally estimated using the LMS algorithm. To implement these algorithm, following steps are described below.

(1) Choose the number of clusters $k$ and input a dataset of $n$ patterns $x=\left[x_{1}, x_{2}, \ldots, x_{n}\right]$. Randomly select the 


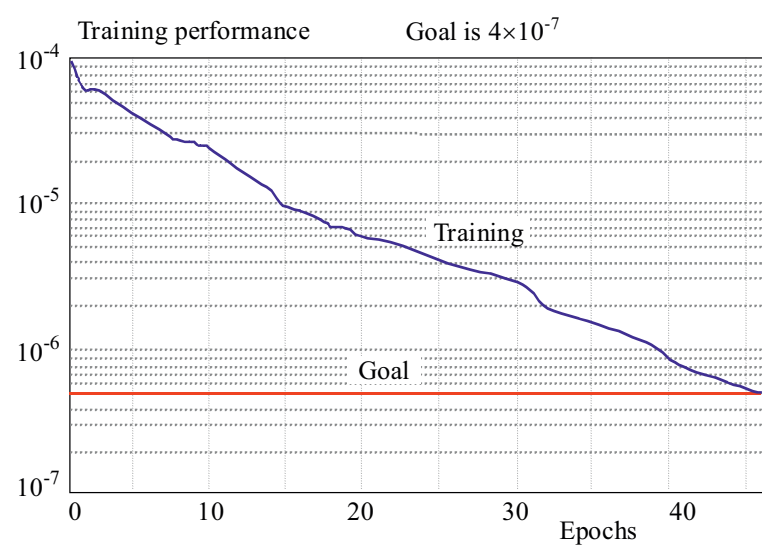

Fig. 4. The training error trajectory

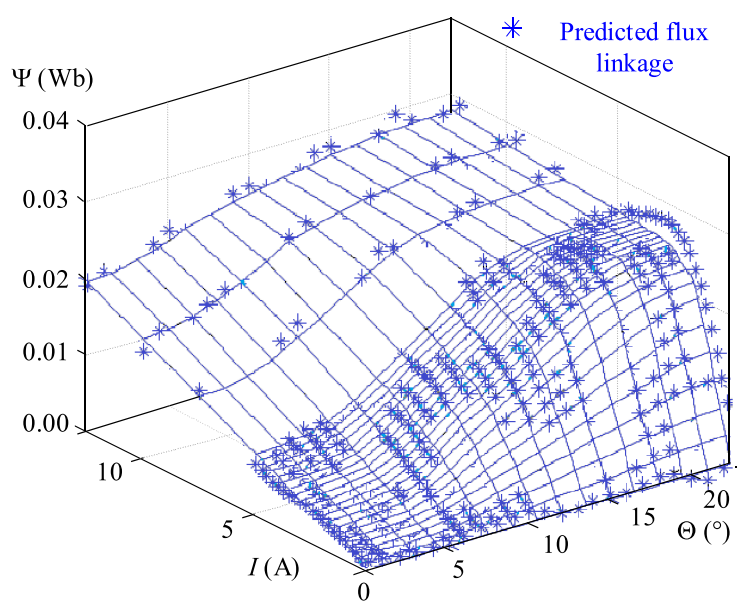

Fig. 5. The flux linkage estimation results

Table 1. The network architecture and training parameters of the offline model

\begin{tabular}{lc}
\hline Number of input variables & 2 \\
Number of output variables & 1 \\
Number of Hidden layer & 1 \\
Number of Neurons in the hidden layer & 30 \\
Goal of Error & $4.5 \times 10^{-7}$ \\
\hline
\end{tabular}

initial candidates for $\mathrm{k}$ cluster centers matrix from the datasets.

(2) Assign each pattern to the nearest cluster using a distance measure. For some set of centers $c=\left[c_{1}, c_{2}, \ldots, c_{n}\right]$, and for each cluster centroid $c_{i}$, we denote the interior of its corresponding cluster as $i\left(x_{n}\right)$, defined as

$$
i\left(x_{n}\right)=\arg \min _{i}\left\|x_{n}-c_{i}\right\|(i=1,2, \ldots, S) .
$$

(3) The RBF centers are learnt using the $k$-means clustering method

$$
c_{i}(k+1)= \begin{cases}c_{i}(k)+\alpha\left[x_{n}(k)-c_{i}(k)\right], & i=i\left(x_{n}\right) \\ c_{i}(k), & i \neq i\left(x_{n}\right)\end{cases}
$$

where, $\alpha$ is the step size, and $0<\alpha<1$.
4 When, $c_{i}(k+1) \approx c_{i}(k)$, step is teminated, otherwise, $k=k+1$ and return to step 1 .

5 The width $\sigma_{i}^{2}$ can be calulated by (9), after the clustering process has converged.

$$
\sigma_{i}^{2}=d_{\max }^{2} / 2 S
$$

where, $d_{\max }$ is the maximum distance between the RBF centers $c_{i} ; S$ is the number of hidden nodes.

6 The LMS algorithm is applied for calibrating the output weights $\boldsymbol{w}=\left[w_{1}, w_{2}, \ldots, w_{n}\right]$, and the adaptation equation is represented by

$$
w(k+1)=w(k)+\eta x(k) e(k)
$$

where $e(k)=d(k)-w^{\top}(k) x(k) ; x(k)$ is the input sample and $d(k)$ is desired output; $\eta$ is the learning-rate parameter.

\subsubsection{Offline modelling of flux linkage characteristics}

As shown in Fig. 3, the offline RBF network based flux linkage model can be considered as a two-input oneoutput input-output nonlinear system. The phase current $i$ and rotor position $\theta$ are defined as the input, and the flux linkage $\Psi$ is defined as the output. The flux linkage characteristics data have been obtained from FEA, which are defined as two inputs one output input-output training pairs.

Generally, based on the training data set, the training process can be performed in Matlab environment simply. However, to meet the requirement of real-time control in DSP platform, the RBF model should be appropriately simplified at the expense of some modelling precisions. In addition, the output weights should distribute uniformly to avoid the output errors that caused by DSP A/D sampling errors, which can be achieved by adjusting the training error goals and the number of the neurons in the hidden layer. In this work, the design of RBF network and selection of optimum training parameters have been evaluated by trial both through simulation and experiments. The network architecture and training parameters obtained are given in Table 1. Based on these parameters, the training processes are performed in Matlab environment. As shown in Figs. 4 and 5, the training error can meet the training goal within 50 epochs and the predicted flux-linkage can well approximate the targets value.

\subsubsection{Online modelling of flux linkage characteristics}

The proposed online-modelling scheme is illustrated in Fig. 6. As shown in the figure, the estimation error between the estimated flux linkage of the offline RBF model and the calculated phase flux linkage is used for 


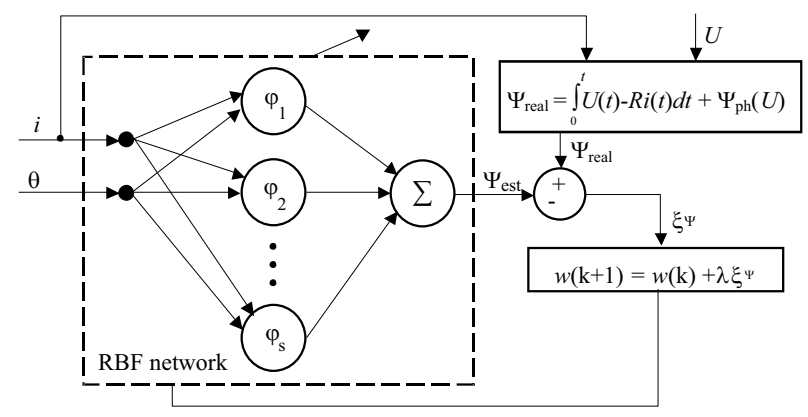

Fig. 6. The principle diagram of the online-modelling scheme
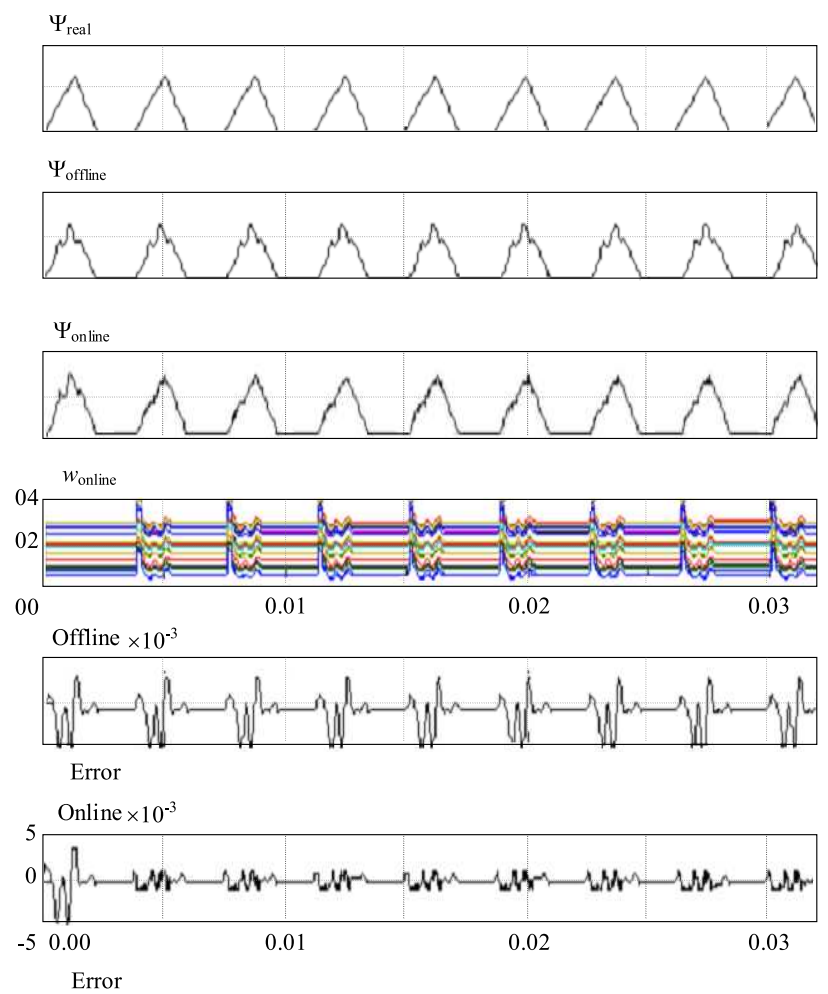

Fig. 7. The simulation results of the offline and online modelling schemes

calibrating the output weights of the offline RBF model. The training algorithm is represented by

$$
\begin{aligned}
w(k+1) & =w(k)+\lambda \times \xi_{\Psi}, \\
\xi_{\Psi} & =\Psi_{\text {real }}-\Psi_{\text {est }}, \\
\Psi_{\text {real }} & =\int_{0}^{t}(U(t)-R i(t)) \mathrm{d} t+\psi_{\mathrm{psi}}(0)
\end{aligned}
$$

where $w$ is the output weight, $k$ is the sample number, and $\lambda$ is the learning rate; $\xi_{\psi}$ is the estimated flux linkage error; $\Psi_{\text {real }}$ is the calculated flux linkage based on the online phase current and voltage; $\Psi_{\text {est }}$ is the estimated flux linkage by the RBF model. As a tradeoff of the accuracy and the training speed, the learning rate $\lambda$ is set as a constant in the experiments and simulation, which equals 0.15 .

\section{SIMULATION AND EXPERIMENTAL VALIDATION}

To evaluate the performance of the proposed modelling schemes, both the simulations and experiments have been performed in a $12 / 8$ structure SRM prototype under the same control conditions. The simulated offline and online modelling results under $2000 \mathrm{rpm}$ are shown in Fig. 7. As can be seen in this figure, the estimation accuracy of the offline model is low. But after applying the online algorithm, the network weights of the output layer are able to be adjusted automatically, and the modelling accuracy can be well improved. Traces 5 and 6 of the figure show the difference.

In order to verify the proposed method, the experimental test-bed was developed. For hardware implementation, a three-phase $12 / 8$ Switched reluctance motor is used as a prototype machine; the power converter is the conventional three phase asymmetric half-bridge converter; the current/voltage sensors and signal modulation circuits are used for measuring the phase current and voltage; the controller was developed based on TMS320F2812 DSP chip, which is a 32-bit fix-point flash DSP, with 150 $\mathrm{MHz}$ clock frequency and 16 channel 12-bit ADC, and two event manager modules for motion control.

Based on the experimental system, the offline and online models are implemented on the DSP2812 controller. The modelling results are shown in Fig. 8 (a) and (b) respectively. As compared to the offline modelling, the estimated flux-l inkage becomes smoother after online adjustment, and the modelling accuracy are greatly improved. As compared with the simulation results, it can be easily find that they are in good agreement. For further verifying the adjust capacity and the control effects of the online modelling scheme, the dynamic reference flux-linkage transient performance of the offline model and online model are tested respectively in the experiments. As shown in Fig. 9 (a), the estimated flux-linkage of the offline model can not track the sudden change of reference flux-linkage. But through online modelling, the output weights of the RBF neural network can be adjusted automatically according to the error of the estimated flux-linkage and the reference flux-linkage. Thus, as shown in Fig. 9 (b), through the self adjustment, the estimated flux-linkage can approach the reference fluxlinkage rapidly.

\section{CONCLUSION}

In conclusion, the RBF neural network based offline and online modelling methods for SRM have been proposed in this paper. The modelling performance of the two kinds of method are evaluated and compared by simulation and experiments. The results demonstrate that the RBF based on-line modelling scheme is a good candidate for SRM modelling, which possess good accuracy, robustness and generality. Furthermore, this work implemented the RBF neural network in experiments successfully, which possess good potential for using in other kinds 

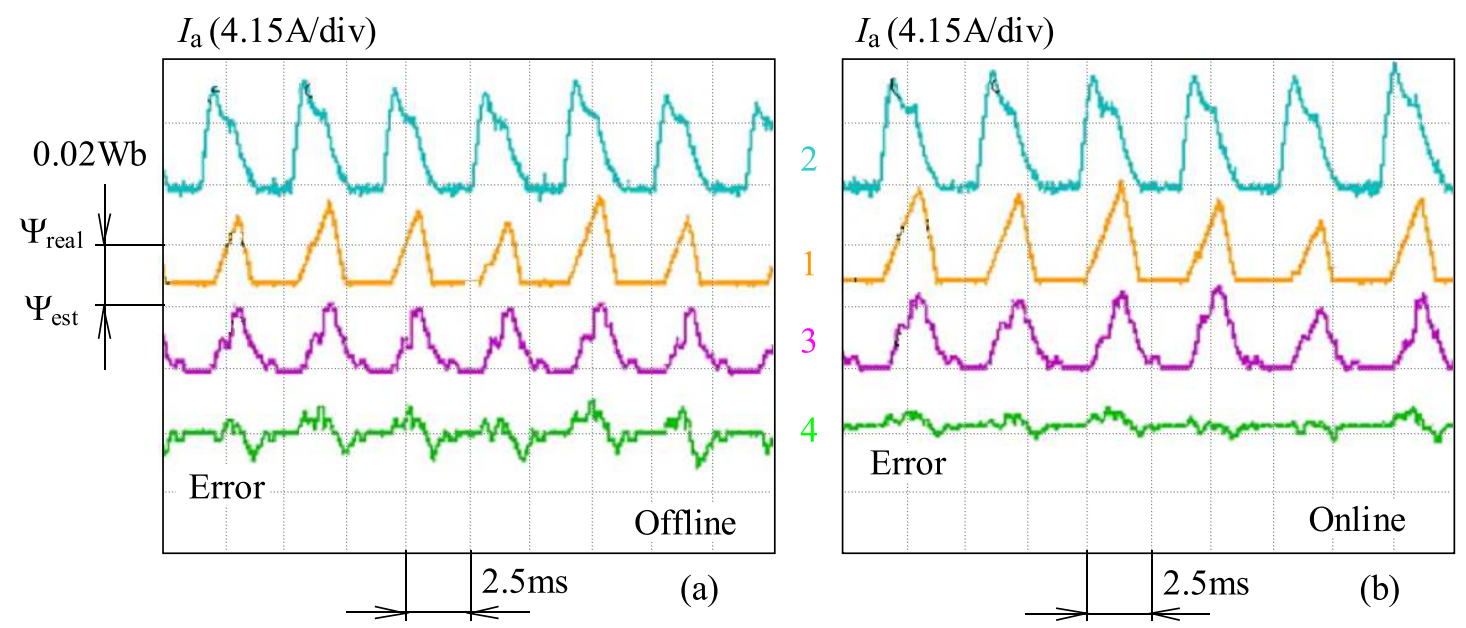

Fig. 8. The experimental results of the offline and online modelling schemes, the result of: (a) - offline, (b) - online modelling scheme
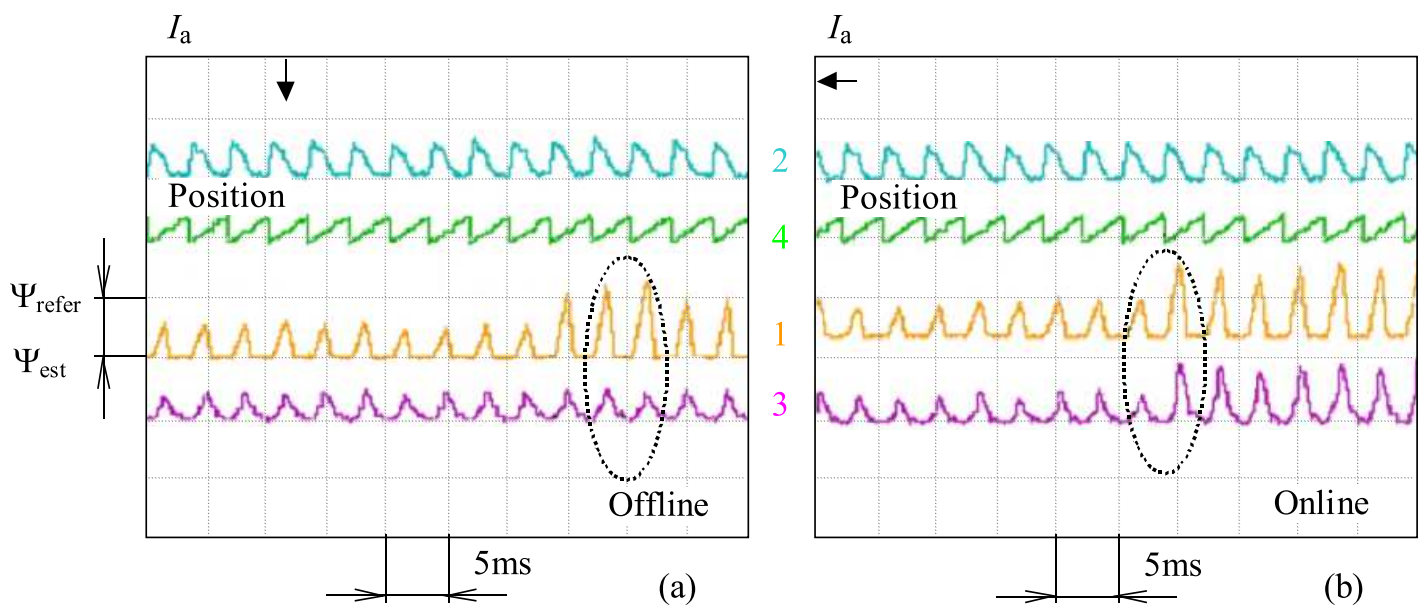

Fig. 9. The experimental results of the offline and online modelling schemes, the result of: (a) - offline, (b) - online modelling scheme

of applications such as parameter identification, function approximation, adaptive control and expert system.

\section{REFERENCES}

[1] CHEN, H. J.-JIANG, D. Q.-YANG, J.-SHI, L. X. : A New Analytical Model for Switched Reluctance Motors, IEEE Transactions on Magnetics 45 (2009), 3107-3113.

[2] MAO, S. H.-DORRELL, D.-TSAI, M. C.: Fast Analytical Determination of Aligned and Unaligned Flux Linkage in Switched Reluctance Motors Based on a Magnetic Circuit Model, IEEE Transactions on Magnetics 45 (2009), 2935-2942.

[3] LYONS, J. P.-MacMINN, S. R.-PRESTON, M. A.: Flux/ Current Methods for SRM Rotor Position Estimation, Conf. Rec. IEEE-IAS Annu. Meeting, 1991, pp. 482-487.

[4] CHEOK, A.-ERTUGRUL, N.: High Robustness and Reliability of Fuzzy Logic Based Position Estimation for Sensorless Switched Reluctance Motor Drives, IEEE Transactions on Power Electronics 15 (2000), 319-334.

[5] MESE, E.-TORREY, D. A. : An Approach for Sensorless Position Estimation for Switched Reluctance Motors using Artificial Neural Networks, IEEE Transactions on Power Electronics 17 (2002), 66-75.

[6] LIN, Z.-REAY, D. S. : Online Modelling for Switched Reluctance Motors using B-Spline Neural Networks, IEEE Transaction Industrial Electronic 54 (2007), 3317-3322.
Received 2 March 2012

Jun Cai was born in Hunan Province, China, in 1982. He received the $\mathrm{PhD}$ degree in electrical engineering in 2012 from Nanjing University of Aeronautics and Astronautics, Nanjing, China. He is now an author of more than 30 peer reviewed journal papers and conference papers, and holds 14 patents. His current research interests include switched reluctance starter/generator, sensorless techniques, linear motor, EV drive system and energy management, and inverter control.

Zhiquan Deng was born in Hubei Province, China, in 1969. He received the BS degree in mechanical engineering from Xian Institute of Metallurgy and Construction Engineering (Renamed as Xian University of Architecture \& Technology since 1994), Xian, China, in 1990, and MS, PhD degrees in Engineering Machinery from Northeastern University, Shenyang, China, in 1993 and 1996, respectively. In 1996, he was a Post-Doctoral at Nanjing University of Aeronautics \& Astronautics, Nanjing, China. In 1998, he joined the Department of Electrical Engineering, Nanjing University of Aeronautics \& Astronautics, Nanjing, China, where he is currently a Professor in the College of Automatic Engineering. He is engaged in research on bearingless motor drive systems, magnetic bearings, super high speed electrical machines. 